\title{
EVALUATION OF THE Y-SHAPED MODIFICATION OF THE TRANSCONJUCTIVAL APPROACH IN OPEN REDUCTION OF ZYGOMATIC MAXILLARY COMPLEX FRACTURE (CLINICAL TRIAL)
}

\author{
Ahmed M. Yassin ${ }^{1 *}$ BDS, Ahmed M. Shaaban ${ }^{2}$ PhD, \\ Marwa G. Noureldin ${ }^{3}$ PhD.
}

\begin{abstract}
INTRODUCTION: Conventional incisions that are commonly used for the exposure of lower eyelid and treatment of Zygomaticomaxillary Complex (ZMC) fractures, includes both transcutaneous and transconjunctival approaches. The decision between the incisions is based on the need for adequate exposure of the fracture site and a desired aesthetically acceptable incision. A "Y” shaped technique was claimed to fulfill both adequate exposure and esthetic outcomes.
\end{abstract}

Aim: This study was conducted to evaluate the Y-shaped modification of the transconjunctival in the management of zygomatic maxillary complex fracture.

MATERIALS AND METHODS: This study was based on seventeen patients with zygomaticomaxillary complex fractures. Post-operative patient evaluation was performed with consideration to intraoperative accessibility, exposure duration, postoperative pain and edema, postoperative wound healing and infection, scarring, and postoperative orbital movement and ocular complications.

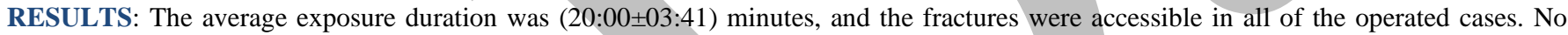
scars were detected in all cases 6 weeks post-operatively. There was a significant difference between post-operative pain status in different intervals ( $\mathrm{p}<0.001)$. A significantly high percentage of cases didn’t have post-ocular complications 15(88.2\%) $(\mathrm{p}<0.001)$.

CONCLUSION: The modification of the transconjunctival approach with a cutaneous Y-shaped incision provides commodious surgical exposure for ZMC fractures.

KEYWORDS: zygomaticomaxillary complex fractures, transconjunctival, preseptal approach.

1 Bachelor of Dental medicine and surgery 2008, Faculty of Dentistry, Alexandria University, Egypt.

2 Professor of Oral and Maxillofacial Surgery, Faculty of Dentistry, Alexandria University, Egypt.

3 Lecturer of Oral and Maxillofacial Surgery, Faculty of Dentistry, Alexandria University, Egypt.

*Corresponding author:

E-mail:_doctor_ai_2@yahoo.com

\section{INTRODUCTION}

The zygomaticomaxillary complex (ZMC) plays a major role in adjusting the midface width and defining the shape and contour of the inferior and lateral orbital borders. It also determines the cheek prominence. It is a tetrapod structure involving the maxilla, frontal bone, temporal bone, and sphenoid bone (1). The treatment of ZMC fractures is challenging due to the affliction of its tetrapod configuration. Thus, improper diagnosis and treatment of these injuries may result in disturbed cosmetic and function (1).

Treatment modalities of ZMC fractures involve observation, closed reduction without fixation or open reduction, and rigid internal fixation (OR/IF) at one or more buttresses. Almost (77 to 94\%) of ZMC fractures are surgically repaired $(2,3)$.
Traditional lower eyelid incisions that are regularly used for the exposure of the zygomatic bone encompass around the infraorbital, subciliary, subtarsal, and transconjunctival approaches. The preference between transcutaneous and transconjunctival incisions must be based on the need for a satisfactory exposure of the fracture site and a desired aesthetically acceptable incision.

Bourquet in 1924 popularized the transconjunctival approach for lower eyelid blepharoplasty (4-6). This approach edges other cutaneous approaches for its hidden orbital incision and decreased risk of ectropion.

Traditional lateral canthotomy is straight in shape. Noval et al proposed a modification to the extended transconjunctival approach where the lateral canthotomy cutaneous incision line is drawn in a Y-shaped manner 
with regular cantholysis of the inferior limb of the lateral canthus tendon (7).

The immense leverage of the lateral canthotomy $\mathrm{Y}$ Modification and cantholysis is the ample presentation of the inferior orbital rim and orbital floor along with the lateral orbital wall and frontozygomatic region (7-9).

Therefore, the aim of this study was to evaluate the $\mathrm{Y}$ modification of the transconjunctival approach in open reduction of the zygomatic maxillary complex fracture.

\section{MATERIAL AND METHODS}

The study was a prospective clinical study. It was conducted on a sample consisting of seventeen patients from those admitted to the Emergency Ward of Alexandria University teaching hospital. The study was conducted after receiving ethical approval from the Alexandria University Faculty of Dentistry's Research Ethics Committee.

\section{Patient's selection}

\section{Eligibility criteria}

\section{a. Inclusion criteria}

- Subjects with zygomaticomaxillary complex (ZMC) fractures that mandate open reduction and internal fixation.

- Patient of age between 20 and 50 years with no gender proclivity.

\section{b. Exclusion criteria}

- Inferior and lateral periorbital regions preexisting lacerations.

- Comminuted fracture with bone loss.

- Fracture line infection.

- Existence of acute or chronic conjunctival diseases.

Preoperative assessment and evaluation

History was collected from all patients. After that radiographic examination through a preoperative computed tomography was taken for each patient preoperatively to assess the degree, severity of fractures and to clarify the type of fracture. (Figure 1)

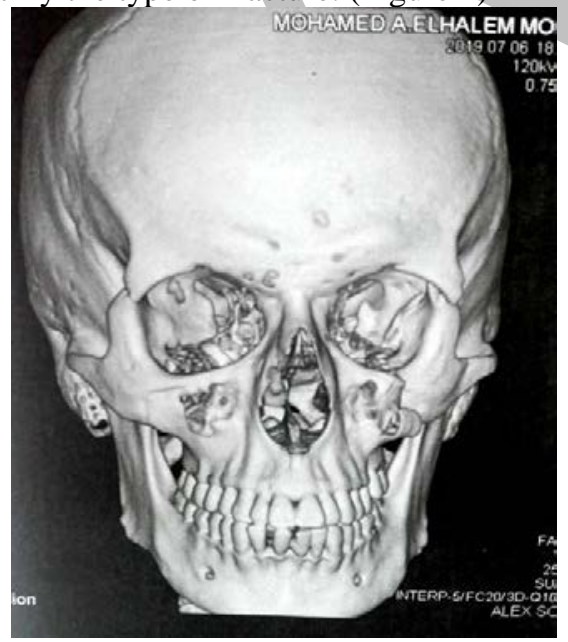

Figure (1): Preoperative.

\section{Surgical procedure}

A Surgical approval was obtained from the anesthesia specialist. Prophylactic antibiotic therapy was administered preoperatively in the form of Cefotaxime 1gm/12 (Cefotax: each vial contains cefotaxime [as sodium salt] $1 \mathrm{gm}$, manufactured by E.I.P.I.C.O) intravenously to prevent postoperative infection. The surgical procedure went in this sequence $(8,9)$ :

1. All patients were operated on using general anesthesia.

2. A forced duction test was performed followed by corneal protectors and a "Y" shaped drawing following a skin crease on the lateral aspect of the lateral canthal angle (Figure 2 A). Another forced duction test was performed at the end of the operation.

3. Subcutaneous anesthesia with epinephrine infiltration was performed below the marked incision.

4. A 3-0 vicryl suture was passed through the tarsus to aid with lid retraction (Figure $2 \mathrm{~B}$ ).

5. The skin incision was made in a Y-shaped manner and it ended with the cutting of the superficial stem of the lateral canthal tendon to separate the tarsal plates

(Figure $2 \mathrm{C}$ ).

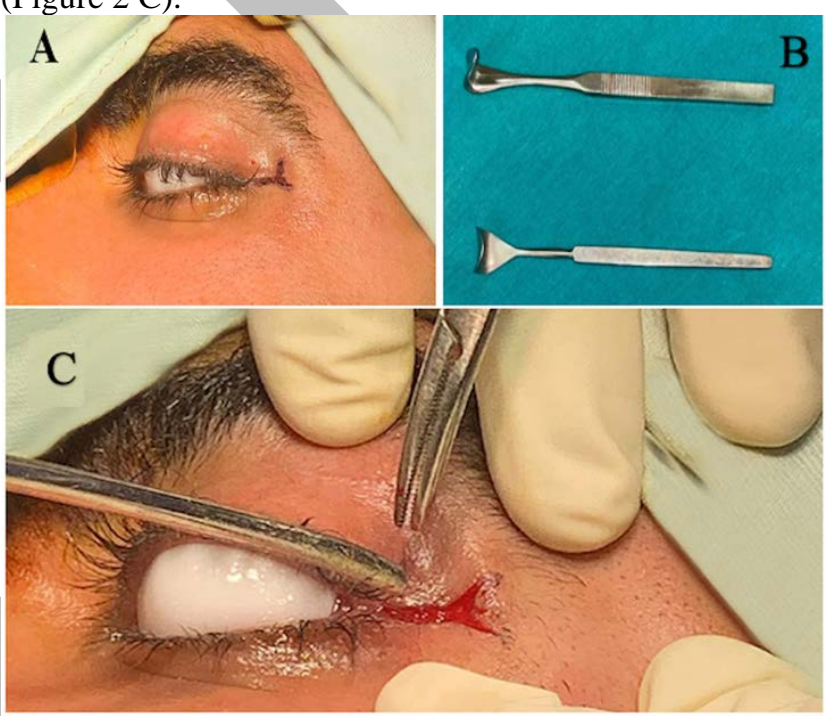

Figure (2): A) intra operative Y marking incision. B) lower eyelid retractor. C) Surgical intraoperative incision

6. After canthotomy, the conjunctiva was exposed and a tunnel beneath the conjunctiva was created using a blunt hemostat toward the medial end of the incision. With one arm of the surgical scissor placed inside the tunnel and the other arm on the outside of the conjunctiva, sectioning of the conjunctiva was performed with attention to maintain a $5 \mathrm{~mm}$ distance down from the lower tarsal plate. Blunt dissection was proceeded in a preseptal plane to reach the infraorbital rim. (Figure $3 \mathrm{~A}$ )

7. Section of the orbital septum was done on the facial aspect of the maxilla, followed by sub-periosteal dissection to denude the infraorbital rim and orbital floor. Consequently, sub-periosteal dissection was continued on the lateral orbital margin to reveal the fracture at the frontozygomatic region. (Figure $3 \mathrm{~B}$ )

8. The superficial $\mathrm{Y}$ was turned into a box by retraction of the incision corners, which expand the working area and grant commodious and concurrent access to 
the lateral orbital wall, and infraorbital rim with the orbital floor in a single approach.

9. A vestibular incision was performed to expose the zygomatic buttress. Fracture reduction and miniplates fixation were implemented according to each case (Figure $3 \mathrm{C}$ ).

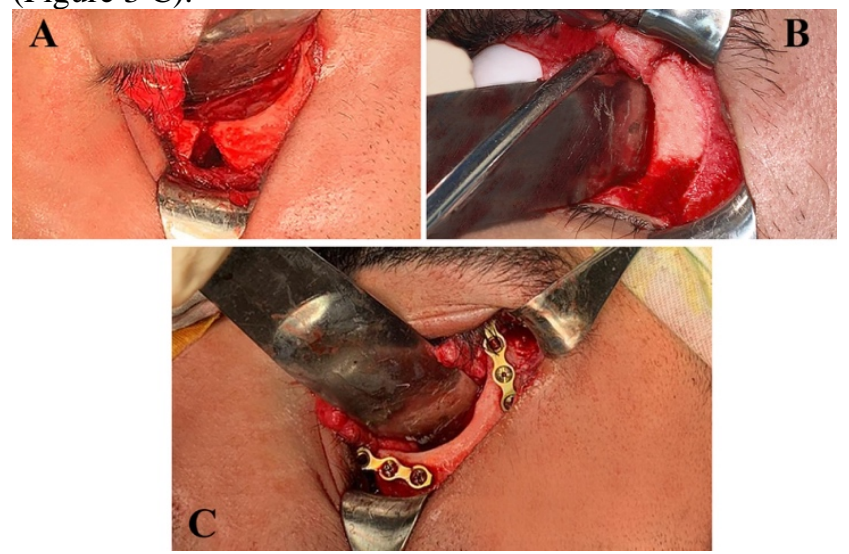

Figure (3): (A) Infra orbital fracture. B) Lateral orbital fracture. C) Reduction and fixation.

10. Wound closure was performed starting with the anterior limb of the lateral canthal ligament and the orbicularis occuli muscle using a 4-0 vicryl suture. The conjunctiva is sutured utilizing a 6-0 vicryl suture. Finally, skin superficial to lateral canthus was sutured to the initial $\mathrm{Y}$ shape utilizing a 6-0 prolene suture (Figure 4).

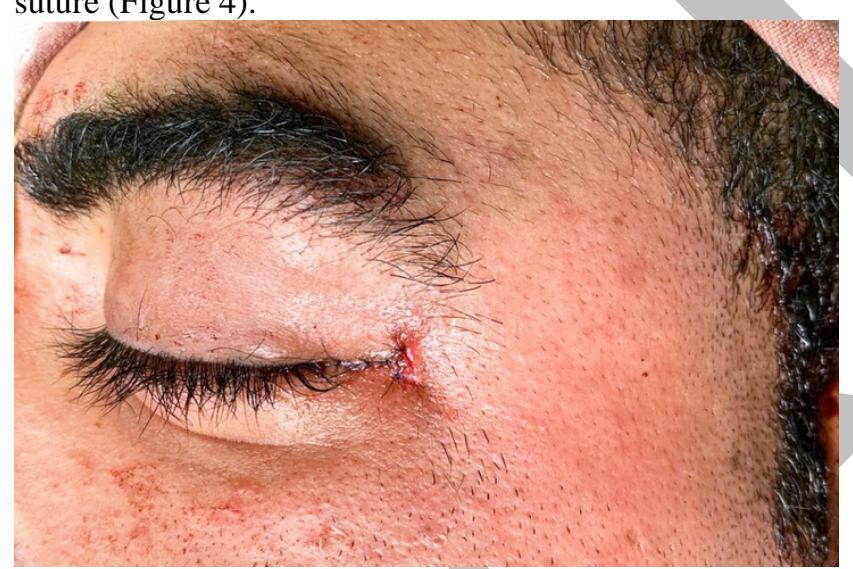

Figure (4): Suturing Y incision.

Post-operative phase

All patients were instructed to apply an ice pack extraorally starting immediately postoperatively for 12 hours. All patients received oral antibiotics in the form of amoxicillin + clavulanate $1 \mathrm{gm}$ twice daily for the next 5 days (Augmentin: amoxicillin 875mg +clavulanic acid 125 mg: GlaxoSmithKline, UK). Analgesics and antiinflammatory medications in the form of Diclofenac potassium (Cataflam: diclofenac potassium $50 \mathrm{mg}$ : Novartis-Switzerland) $50 \mathrm{mg}$ every night for 5 days. All patients were instructed to rinse their mouth using chlorhexidine (Hexitol: chlorhexidine125mg/100ml, concentration0.125\%: Arabic drug company, ADCO.) antiseptic mouth wash in cases where an additional vestibular maxillary intraoral incision was needed. Instruction of soft, fully liquid, high protein, high-calorie diet was given for all patients for weeks postoperatively. Sutures were removed after 10 days.

\section{Follow Up Phase}

\section{a. Clinical evaluation}

Accessibility to the fracture site and exposure duration was recorded and noted during the operation. Patients were evaluated and follow up after 24 hours, 48 hours, 1 week, 3 weeks, 4 weeks, 6 weeks, and 3 months. Clinical assessment of postoperative pain, edema, postoperative scar esthetic appearance, sensory nerve function, and wound healing. Postoperative ocular complications; such as ectropion, entropion, corneal damage, restriction of ocular mobility, or chemosis were assessed and reported. (Figure 5)

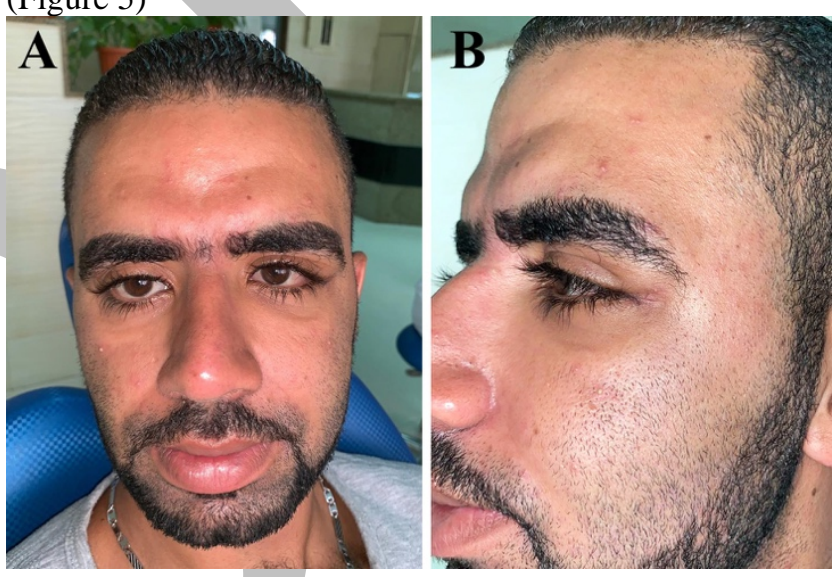

Figure (5): A\&B Clinical Follow up.

b. Radiographic evaluation

Immediate postoperative computed tomography was obtained to assess the adequacy of fracture line reduction.

Statistical analysis

Frequencies (n) and percentages (\%) were used to present categorical data, which was analyzed using Fisher's exact test.

Normality testing for the Quantitative data was performed using Kolmogorov-Smirnov and Shapiro-Wilk tests and they were presented as mean and standard deviation values. Nonparametric data were analyzed utilizing Freidman's test followed by Dunn's post hoc test. The significance level was set at $\mathrm{P} \leq 0.05$ for all tests and $\mathrm{p}$ values were corrected for multiple comparisons using Bonferroni correction. Statistical analysis was performed with IBM ${ }^{\circledR}$ SPSS ${ }^{\circledR}$ (SPSS Inc., IBM Corporation, NY, USA) Statistics Version 25 for Windows.

\section{RESULTS}

\section{a. Demographic data}

This study was performed on 17 patients with a mean age of $31.82 \pm 9.23$ years. The population sample included a significantly higher percentage of males $14(82.4 \%)$ in comparison to 3 (17.6\%) females.

There was a significant difference in the distribution of different etiological causes $(p<0.001)$. The most prevalent cause of fracture was road traffic accidents (RTA) 11 $(64.7 \%)$ while other causes like being in a fight or falling 
from heights only occurred in 3 (17.6\%) cases each (Table $1)$.

Table (1): Frequencies (n) and percentages (\%) of fracture etiology

\begin{tabular}{|l|c|c|c||}
\hline $\begin{array}{l}\text { Etiology of } \\
\text { fracture }\end{array}$ & $\mathbf{n}$ & $\%$ & p-value \\
\cline { 1 - 3 } RTA & $11 \mathrm{~A}$ & $64.7 \%$ & \\
\cline { 1 - 3 } Fight & $3 \mathrm{~B}$ & $17.6 \%$ & \multirow{2}{*}{$<\mathbf{0 . 0 0 1 *}$} \\
\cline { 1 - 3 } $\begin{array}{l}\text { Falling from a } \\
\text { height }\end{array}$ & $3 \mathrm{~B}$ & $17.6 \%$ & \\
\hline
\end{tabular}

Different superscript letters indicate a statistically significant difference within the same vertical column

*; significant $(\mathrm{p} \leq 0.05) \mathrm{ns}$; non-significant $(\mathrm{p}>0.05)$

b. Accessibility of fracture site and exposure duration

The fractures were accessible in all of the operated cases and the average exposure duration was (20:00 $\pm 03: 41)$ minutes.

\section{c. Clinical Data}

A statistically significant difference was observed for the status of postoperative edema at different follow-up periods $(\mathrm{p}<0.001)$. At 24 hours, cases were nearly divided between having moderate to severe $9(52.9 \%)$ and severe $8(47.1 \%)$ edema. After 48 hours, the majority of the cases either had mild to moderate or moderate edema 7(41.2\%). While after 1 week all cases had mild edema. No scars were detected in all cases 6 weeks post-operatively.

There was a significant difference between post-operative pain status in different intervals. The highest record of (VAS) was observed after 24 hours $(8.35 \pm 0.86)$, which continued to decrease over time with a value of $(5.76 \pm 1.35)$ after 48 hours and then $(0.65 \pm 0.76)$ after 1 week. Post hoc pairwise comparisons showed values measured at different intervals to be significantly different from each other $(\mathrm{p}<0.001)$.

A significantly high percentage of cases felt normal cheek innervation and infraorbital nerve function 14(82.4\%) $(p<0.001)$. A significantly higher percentage of cases didn't have post-ocular complications 15(88.2\%) $(\mathrm{p}<0.001)$. Only 2 cases reported postoperative lower eyelid ectropion and increase in the amount of sclera show in comparison to the contralateral side. Both cases regained normal lower eyelid appearance by the end of the follow up period without the need for a suspensory sutures (Table 2,3).

Table (2): Frequencies (n) and percentages (\%) for status of post-ocular complications.

\begin{tabular}{|l|c|c|c|}
\hline $\begin{array}{l}\text { Post-ocular } \\
\text { complications }\end{array}$ & N & $\%$ & p-value \\
\hline
\end{tabular}

\begin{tabular}{|l|c|c|c|}
\hline $\begin{array}{l}\text { Post-ocular } \\
\text { complications }\end{array}$ & $\mathbf{N}$ & $\mathbf{\%}$ & \multirow{2}{*}{ p-value } \\
\cline { 1 - 3 } Normal & $15 \mathrm{~A}$ & $88.2 \%$ & \multirow{2}{*}{$<0.001 *$} \\
\cline { 1 - 3 } Slight ectropion & $1 \mathrm{~B}$ & $5.9 \%$ & \multirow{2}{*}{ * } \\
\cline { 1 - 3 } Ectropion & $1 \mathrm{~B}$ & $5.9 \%$ & \\
\hline
\end{tabular}

Different superscript letters indicate a statistically significant difference within the same vertical column

*; significant $(\mathrm{p} \leq 0.05) \mathrm{ns}$; non-significant $(\mathrm{p}>0.05)$

Table (3):. Mean and standard deviation (SD) values of Visual Analogue Scale (VAS).

\begin{tabular}{||c|c|c|c|}
\hline \multicolumn{3}{|c|}{ VAS (Mean \pm SD) } & p-value \\
\hline \multirow{2}{*}{$\mathbf{2 4}$ hours } & $\mathbf{4 8}$ hours & $\mathbf{1}$ week & \multirow{2}{*}{$<\mathbf{0 . 0 0 1 *}$} \\
\cline { 1 - 2 } $8.35 \pm 0.86$ & $5.76 \pm 1.35$ & $0.65 \pm 0.76$ & \\
\hline
\end{tabular}

Different superscript letters indicate a statistically significant difference within the same vertical column

*; significant $(\mathrm{p} \leq 0.05) \mathrm{ns}$; non-significant $(\mathrm{p}>0.05)$

\section{DISCUSSION}

The zygomatic bone possesses an innate bizarre architecture and takes part in forming both the horizontal and vertical buttress of the facial skeleton. Proper anatomical reconstruction of the zygomatic processes along with the orbital floor is the optimal outcome of the ZMC fracture management $(1,10)$.

The different location of the bone segments dictates multiple and different incision line location to ensure adequate exposure and working manipulation (5). Transconjunctival approach with canthotomy and cantholysis bring upon exemplary exposure to the orbital floor and inferior rim (7-8). Moreover; cutaneous modification of the straight lateral canthotomy incision into a Y-shaped incision line gives adequate and coexisting access to the lateral orbital wall without the need for a secondary approach (7-9). Hence the aim of this work was to examine the use of the transconjunctival approach with Y-modification for the simultaneous visualization, reduction, and fixation of the infraorbital rim and the lateral orbital margin.

Mean age of $31.82 \pm 9.23$ years and a male to female ratio of 4.67:1 was the demographic outcome of this study. A similar ratio was reported by Ellis et al, which reported an average age of 29.1 years. A common consensus in the literature that a prevalence of young adults dominates in ZMC fractures (70-80\%). The most reported fracture etiological factor was road traffic accidents (RTA) (64.7\%, $\mathrm{n}=11$ ). Devi et al (2015) reported a higher, $80 \%$, of cases with RTA as their causative factor. The increased load on the roads, along with the poor and unattained road traffic laws, poor conditions of the roads and vehicles, and traveling without using safety precautions are all common 
findings in developing countries, which may attribute to the high rates of RTA (11-13).

The average fracture line exposure time was recorded from the start of the incision till the full visualization of the operative field. This time is independent of the fracture reduction time and total operation time. The average exposure duration was 20:00 \pm 03:41 minutes. This recorded interval was nearly 2 min longer than the average reported by Devi et al. (2015), which was 18.80 minutes, giving a comparable outcome (11). Devi et al. (2015) reported a self-explanatory statement that a single incision transconjunctival Y-modification technique took statistically significant less time than the combined subtarsal lateral eyebrow approach, where 22.8 minutes was reported (11).

The additional Y-cutaneous modification shows compatible records with the transconjunctival with lateral canthotomy approach. Girish et al the reported 18.9 minutes while utilizing a transconjunctival approach with lateral canthotomy. A similar duration was reported by Holtmann et al. (20 min) and Subramanian et al. (22 min) for transconjunctival with lateral canthotomy approach $(14,15)$.

All of the patients reported severe postoperative edema reported in the first postoperative period, where all turned into mild edema at the second postoperative period after one week. There is a lack of appreciation for the physiology of orbital lymphatic drainage. Dickinson and Gausas (2006) utilized the recent advances in vascular markers to determine the main draining ports for the orbital tissues, extraocular muscles, and anterior orbital fat. They reported that the superficial and deep lymphatic drainage of both eyelids assembles at the canthus lymphatic vessels, which causes the prolonged time required for drainage (16).

Shoukath et al. (2017) stated that the superficial lymphatic drainage is susceptible to damage during incisions and dissection in the lateral canthus area, where both the superficial and deep lymphatic drains mingle, which may be culpable for postoperative chemosis and edema. The performance of lateral canthotomy is concomitant with severe early postoperative periorbital edema. This is usually a normal and expected complication that will dissipate promptly if the patients strictly adhere to the postoperative instruction. Furthermore; the avoidance of orbital septum incorporation in the incision would result in a more patent lymphatic drainage which may lessen the degree of postoperative periorbital edema (17).

A statistically significant decrease in the value of the reported pain using VAS was reported across the followup period. This was in correlation with the decrease in the amount of periorbital edema $(16,17)$.

Checking the functional outcome of the infraorbital nerve was performed and only three cases reported subjective transient paraesthesia in the course of the nerve during the first two follow-up periods. All of the patients regained their normal sensation by the end of the observational period. A significantly high percentage of cases felt normal 14(82.4\%) $(\mathrm{p}<0.001)(8,9)$
Only one case reported postoperative cutaneous wound dehiscence at the first postoperative period, which subsided by wound care and debridement in the second follow-up period. At the end of the six weeks, no cutaneous scar was detected in all of the study cases. A similar outcome was reached by several other authors utilizing the Y-modification for the conjunctival approach $(8,9,11)$.

The transconjunctival mucous membrane approach with its inconspicuous hidden incision grants operational working access for a diverse number of procedures without enlarging the originally planned incision. The adoption of the transconjunctival approach tends to yield sufficient orbital skeleton exposure while at the same time scaledown the odds of scarring. The routinely performed lateral canthotomy incision breed a mere cutaneous wound, which heal adequately and promptly without any objectable scarring. The Y-cutaneous modification incision lines rest in the natural skin crow's feet skin crease, thus yielding in an inconspicuous scar with a pleasing cosmetic result.

In all of the 17 cases included in this study, the cutaneous Y-modification gave the operator profound access to inferior and lateral orbital rims with no need for an additional lateral eyebrow incision. The early reports of the Y modifications come in accordance with our findings, where sufficient exposure was obtained Martinez et al. (2012), in the 24 subjects, and by Rajkumar et al. (2016), in 10 cases, $(8,9)$. This ample exposure was attained by a superficial portion of the Lateral Canthal Tendon (LCT) canthotomy, along with cantholysis of the deep portion of the LCT. Martinez et al. (2012) utilized a similar technique in cutting the deep portion of the LCT for greater exposure. However; Rajkumar et al. (2016) announced a different technique with only a superficial canthotomy needed. Rajkumar et al. (2016) noted sorts of hardship in the placement of the uppermost screws in the plate along the frontozygomatic suture, and they recommended a more adequate subperiosteal dissection and retraction so that the box created by the Y-incision can be moved further along the lateral orbital rim $(8,9)$.

The utilized technique in this study gave commodious exposure with minimal retraction pressure on the wound edges. On the other hand; this technique entitles the operator for a meticulous reconstruction of the LCT during closure to avert any canthal asymmetry lid mal-positioning $(8,9)$. Ilankovan et al conducted that simultaneous access to the inferior and lateral orbital rims gave this technique a vast dominance when compared to any other approach (18).

Out of the 17 included cases, only two cases (11.8\%) reported postoperative ectropion, with one of them showing a minor scleral show. A significantly higher percentage of cases didn't have post-ocular complications $(\mathrm{p}<0.001)$. A Similar complication percentage was reported by Novelli et al. (2011) (10\%) in their case series of fifty-six cases. While a higher $16.7 \%$ complication percentage was stated by Martinez et al. (2012), and 20\% by Rajkumar et al. (2016). Minor scleral show was the only reported postocular complication, which did not 
represent a clinical problem for any of the affected patients (7-9).

Devi et al. (2015) performed a comparative study between the transconjunctival approach with Y-modification and the double lateral brow and subtarsal incisions. They reported no ectropion after the transconjunctival approach, in comparison to $7.7 \%$ with the subtarsal incisions (11). A plethora of studies postulates that a reduction in lower eyelid retraction occurs with the utilization of the transconjunctival approach decreases the incidence of lower eyelid retraction (19). A meta-analysis conducted in 2009 for the incidence of eyelid malposition after trauma management concluded that transconjunctival alternative reported the least incidence of eyelid malposition (5.1\%; ectropion, $4.7 \%$; entropion, $0.48 \%$ ) when compared to the transcutaneous ones. Ridgway et al. (2009) endorsed the use of transconjunctival incisions with canthotomy and tarsorrhaphy suture (20). Manson et al quoted that ectropion occurs inversely to the experience of the operator (21).

On the other hand; this approach comes with several difficulties, such as it compels manipulation of the conjunctival surfaces, composing the ocular globe, and placing it in a susceptible setting to injury. This may be complicated by the fact that the conjunctiva is a thoroughly unparalleled tissue that has few acceptable options for reconstruction. Furthermore; LCT repositioning is a challenging procedure that necessitates experience and professional operational skills $(8,9)$.

Within the limitations of this study and by analyzing its outcomes, it may be assumed that the utilization of a Ymodification for in the management of ZMC fractures is a peerless surgical approach with plentiful exposure without the need of an additional incision line and provides exemplary esthetic outcomes. It is however a technically demanding approach that requires profound anatomical knowledge and surgical expertise with meticulous handling of the tissues.

\section{CONCLUSIONS}

Despite that it is a technique sensitive approach that requires surgical skills and strict attention to anatomical guidance, the use of the $\mathrm{Y}$-modification with the transconjunctival approach in the management of ZMC fractures is an excellent surgical approach with sufficient exposure without the need for a second incision line and provides excellent esthetic outcomes

CONFLICT OF INTEREST

The authors declare that they have no conflicts of interest FUNDING STATEMNET

This research received no funding.

\section{REFERENCES}

1. Lee EI, Mohan K, Koshy JC, Hollier LH Jr. Optimizing the surgical management of zygomaticomaxillary complex fractures. Semin Plast Surg. 2010;24:389-97.

2. Waheed El-Anwar M, Elsheikh E, Sweed AH, Ezzeldin N. Electromyography assessment in zygomaticomaxillary complex fractures. Oral Maxillofac Surg. 2015;19:375-9.

3. Meslemani D, Kellman RM. Zygomaticomaxillary complex fractures. Arch Facial Plast Surg. 2012;14:62-6.

4. Ishida K. Evolution of the surgical approach to the orbitozygomatic fracture: From a subciliary to a transconjunctival and to a novel extended transconjunctival approach without skin incisions. J Plast Reconstr Aesthet Surg. 2016;69:497-505.

5. Gomes PP, Passeri LA, Barbosa JR. A 5-year retrospective study of zygomatico-orbital complex and zygomatic arch fractures in Sao Paulo State, Brazil. J Oral Maxillofac Surg. 2006;64:63-7.

6. Bourguet J. Les hernies graisseuses de l'orbite: Notre traitement chirurgical. Bull Acad Med (Paris). 1924;92.

7. Novelli G, Ferrari L, Sozzi D, Mazzoleni F, Bozzetti A. Transconjunctival approach in orbital traumatology: a review of 56 cases. J Craniomaxillofac Surg. 2011;39:266-70.

8. Martinez AY, Bradrick JP. Y modification of the transconjunctival approach for management of zygomaticomaxillary complex fractures: a technical note. J Oral Maxillofac Surg. 2012;70:97-101.

9. Rajkumar K, Mukhopadhyay P, Sinha R, Bandyopadhyay TK. ' $Y$ ' Modification of the Transconjunctival Approach for Management of Zygomatic Complex Fractures: A Prospective Analysis. J Maxillofac Oral Surg. 2016;15:45-51.

10. Marinho R, Maia B. Management of Fractures of the Zygomaticomaxillary Complex. Oral Maxillofacial Surg Clin N Am. 2013;25:617-36.

11. Devi RS. Comparison of $y$ - modification of transconjunctival approach versus subtarsal lateral eyebrow approach for zygomaticomaxillary complex fractures: A Prospective study.M.Sc. Thesis. Oral and Maxillofacial Surgery department, Tamilnadu Government Dental College and Hospital, Chennai. 2015

12. Ellis E, Reddy L. Status of the internal orbit after reduction of zygomaticomaxillary complex fractures. J Oral Maxillofac Surg. 2004;62:27583.

13. Gopalakrishnan S. A public health perspective of road traffic accidents. J Family Med Prim Care. 2012;1:144-50.

14. Holtmann B, Wray RC, Little AG. A randomized comparison of four incisions for orbital fractures. Plast Reconstr Surg. 1981;67:731-7.

15. Subramanian B, Krishnamurthy S, Suresh Kumar P, Saravanan B, Padhmanabhan M. Comparison of various approaches for exposure of infraorbital rim fractures of zygoma. J Maxillofac Oral Surg. 2009;8:99-102. 
16. Dickinson AJ, Gausas RE. Orbital lymphatics: do they exist? Eye (Lond). 2006;20:1145-8.

17. Shoukath S, Taylor GI, Mendelson BC, Corlett RJ, Shayan R, Tourani SS, et al. The Lymphatic Anatomy of the Lower Eyelid and Conjunctiva and Correlation with Postoperative Chemosis and Edema. Plast Reconstr Surg. 2017;139:628e-37e.

18. Ilankovan V. Transconjunctival approach to the infraorbital region: a cadaveric and clinical study. Br J Oral Maxillofac Surg. 1991;29:169-72.

19. Appling WD, Patrinely JR, Salzer TA. Transconjunctival approach vs subciliary skinmuscle flap approach for orbital fracture repair. Arch Otolaryngol Head Neck Surg. 1993;119:1000-7.
20. Ridgway EB, Chen C, Colakoglu S, Gautam S, Lee BT. The incidence of lower eyelid malposition after facial fracture repair: a retrospective study and meta-analysis comparing sub- tarsal, subciliary, and transconjunctival incisions. Plast Reconstr Surg. 2009; 124:157886.

21. Manson PN, Crawley WA, Yaremchuk MJ, Rochman GM, Hoopes JE, French JH Jr. Midface fractures: advantages of immediate extended open reduction and bone grafting. Plast Reconstr Surg. 1985;76:1-12.

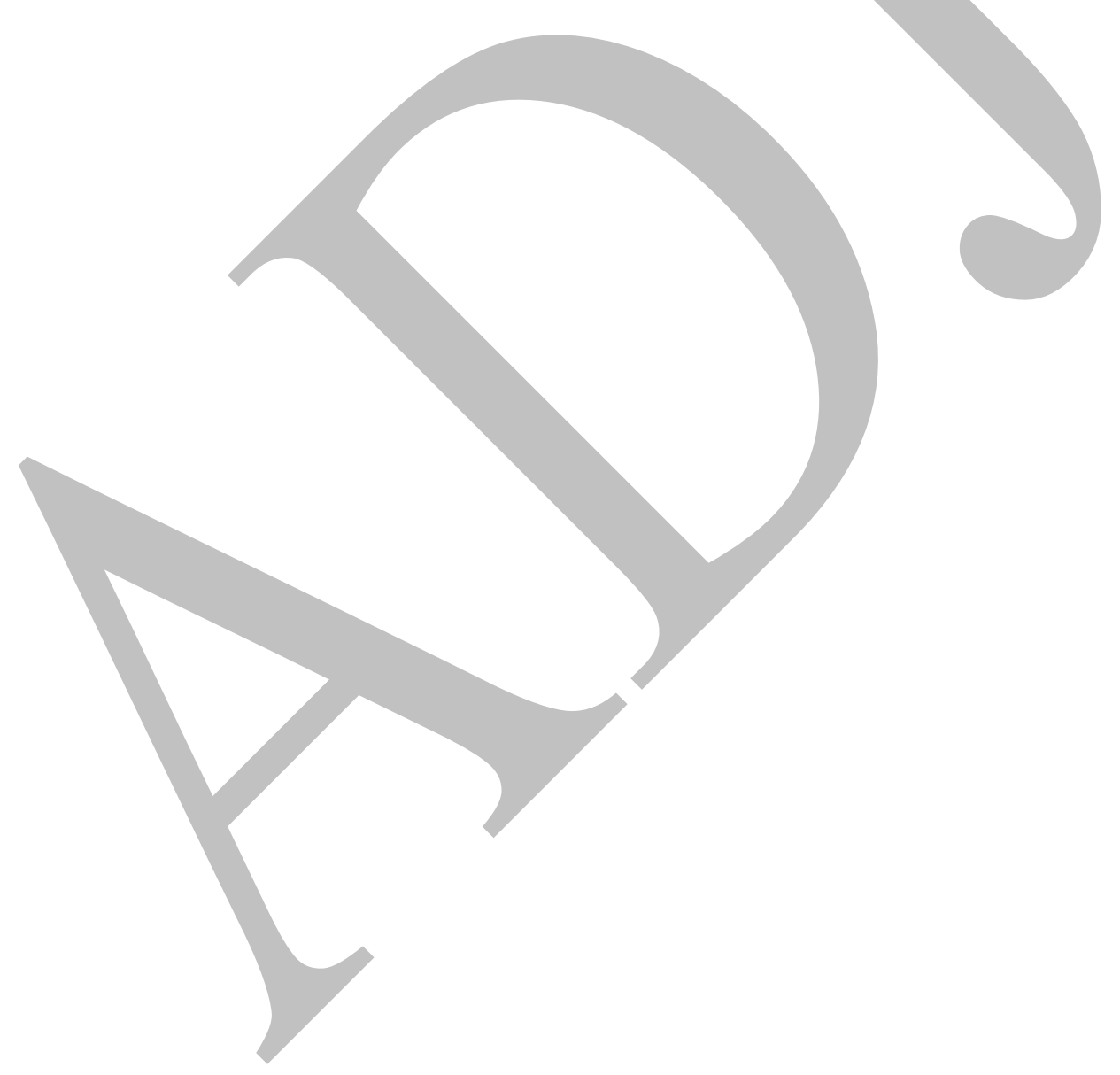

